\title{
Exact Solutions and Conservation Laws of a Two-Dimensional Integrable Generalization of the Kaup-Kupershmidt Equation
}

\author{
Abdullahi Rashid Adem and Chaudry Masood Khalique \\ International Institute for Symmetry Analysis and Mathematical Modelling, Department of Mathematical Sciences, \\ North-West University, Mafikeng Campus, Private Bag X 2046, Mmabatho 2735, South Africa
}

Correspondence should be addressed to Chaudry Masood Khalique; masood.khalique@nwu.ac.za

Received 14 November 2012; Accepted 25 November 2012

Academic Editor: Fazal M. Mahomed

Copyright ( 2013 A. R. Adem and C. M. Khalique. This is an open access article distributed under the Creative Commons Attribution License, which permits unrestricted use, distribution, and reproduction in any medium, provided the original work is properly cited.

\begin{abstract}
We study a two-dimensional integrable generalization of the Kaup-Kupershmidt equation, which arises in various problems in mathematical physics. Exact solutions are obtained using the Lie symmetry method in conjunction with the extended tanh method and the extended Jacobi elliptic function method. In addition to exact solutions we also present conservation laws which are derived using the multiplier approach.
\end{abstract}

\section{Introduction}

The theory of nonlinear evolution equations (NLEEs) has made a substantial progress in the last few decades. Aspects of integrability of these equations have been studied in detail as it is evident from many research papers published in the literature. In many cases, exact solutions are required as numerical methods are not appropriate. Exact solutions of NLEEs arising in fluid dynamics, continuum mechanics, and general relativity are of considerable importance for the light they shed into extreme cases which are not susceptible to numerical treatments. However, finding exact solutions of NLEEs is a difficult task. In spite of this, many new methods have been developed recently that are being used to integrate the NLEEs. Among them are the inverse scattering transform [1], the Hirota's bilinear method [2], the homogeneous balance method [3], the auxiliary ordinary differential equation method [4], the He's variational iteration method [5], the sine-cosine method [6], the extended tanh method [7], the Lie symmetry method [8], and so forth.

In this paper we study a two-dimensional integrable generalization of the Kaup-Kupershmidt equation $[9,10]$

$$
\begin{aligned}
u_{t} & +u_{x x x x x}+\frac{25}{2} u_{x} u_{x x}+5 u u_{x x x}+5 u^{2} u_{x} \\
& +5 u_{x x y}-5 \partial_{x}^{-1} u_{y y}+5 u u_{y}+5 u_{x} \partial_{x}^{-1} u_{y}=0
\end{aligned}
$$

which arises in various problems in many areas of theoretical physics. The above equation arises as special reduction of integrable nonlinear systems $[11,12]$. It should be noted that the Zakharov-Manakov delta dressing method was used to obtain soliton and periodic solutions of (1) [11, 12]. The purpose of this paper is twofold. Firstly, we will use Lie symmetry method along with the extended tanh method and the extended Jacobi elliptic function method to obtain new exact solutions of (1). Secondly, conservation laws will be derived for the two-dimensional integrable generalization of the Kaup-Kupershmidt equation (1).

In the past few decades, the Lie symmetry method has proved to be a versatile tool for solving nonlinear problems described by the differential equations arising in mathematics, physics, and in many other scientific fields of study. For the theory and application of the Lie symmetry method see, for example, $[8,13,14]$.

In the study of the solution process of differential equations (DEs), conservation laws play a central role. It is a well known fact that finding the conservation laws of DEs is often the first step towards finding the solution [14] of the DEs. Extensive use of conservation laws has appeared in the literature, for example, in studying the existence, uniqueness, and stability of solutions to nonlinear partial differential equations [15-17], the use of numerical methods $[18,19]$, and 
finding exact solutions of some nonlinear partial differential equations [20-22].

To study the two-dimensional integrable generalization of the Kaup-Kupershmidt equation (1) we first introduce a new dependent variable $v$ and set $v=\partial_{x}^{-1} u_{y}=\int u_{y} d x$. This allows us to remove the integral terms from the equation and replace (1) by a system

$$
\begin{gathered}
u_{t}+u_{x x x x x}+\frac{25}{2} u_{x} u_{x x}+5 u u_{x x x}+5 u^{2} u_{x} \\
+5 u_{x x y}-5 v_{y}+5 u v_{x}+5 u_{x} v=0 \\
u_{y}-v_{x}=0
\end{gathered}
$$

The outline of the paper is as follows. In Section 2, we first find the Lie point symmetries of the system (2a) and (2b) using the Lie algorithm. These Lie point symmetries are then used to transform the system (2a) and (2b) to a system of ordinary differential equations (ODEs). The extended tanh method and extended Jacobi elliptic function method are applied to the system of ordinary differential equations and as a result we obtain the exact explicit solutions of our two-dimensional integrable generalization of the Kaup-Kupershmidt equation (1). In Section 3, we construct conservation laws for (1) using the multiplier method [23]. Finally, concluding remarks are presented in Section 4.

\section{Exact Solutions of $(2 \mathbf{a})$ and $(2 \mathbf{b})$}

The symmetry group of the system (2a) and (2b) will be generated by the vector field of the form

$$
\begin{aligned}
X= & \xi^{1}(t, x, y, u, v) \frac{\partial}{\partial t}+\xi^{2}(t, x, y, u, v) \frac{\partial}{\partial x} \\
& +\xi^{3}(t, x, y, u, v) \frac{\partial}{\partial y}+\eta^{1}(t, x, y, u, v) \frac{\partial}{\partial u} \\
& +\eta^{2}(t, x, y, u, v) \frac{\partial}{\partial v} .
\end{aligned}
$$

Applying the fifth prolongation, $\mathrm{pr}^{(5)} X,[8]$ to (2a) and (2b) results in an overdetermined system of linear partial differential equations. The general solution of the overdetermined system of linear partial differential equations is given by

$$
\begin{gathered}
\xi^{1}=5 F_{2}(t)+5 y F_{3}^{\prime}(t)+5 y^{2} F_{1}^{\prime \prime}(t)+50 x F_{1}^{\prime}(t), \\
\xi^{2}=150 y F_{1}^{\prime}(t)+75 F_{3}(t), \\
\xi^{3}=250 F_{1}(t), \\
\eta^{1}=5 F_{3}^{\prime}(t)+10 y F_{1}^{\prime \prime}(t)-100 u F_{1}^{\prime}(t), \\
\eta^{2}=-5 u F_{3}^{\prime}(t)+y F_{3}^{\prime \prime}(t)+F_{2}^{\prime}(t)-200 v F_{1}^{\prime}(t) \\
+(10 x-10 y u) F_{1}^{\prime \prime}(t)+y^{2} F_{1}^{\prime \prime \prime}(t),
\end{gathered}
$$

where $F_{1}(t), F_{2}(t)$, and $F_{3}(t)$ are arbitrary functions of $t$. We confine the arbitrary functions to be of the form $F_{1}(t)=$
$C_{1} t+C_{2}, F_{2}(t)=C_{3} t+C_{4}, F_{3}(t)=C_{5} t+C_{6}$, where $C_{1}, \ldots, C_{6}$ are arbitrary constants. Consequently, we have the six-dimensional Lie algebra spanned by the following linearly independent operators:

$$
\begin{gathered}
\Gamma_{1}=\frac{\partial}{\partial t}, \\
\Gamma_{2}=\frac{\partial}{\partial x}, \\
\Gamma_{3}=\frac{\partial}{\partial y}, \\
\Gamma_{4}=5 t \frac{\partial}{\partial x}+\frac{\partial}{\partial v}, \\
\Gamma_{5}=y \frac{\partial}{\partial x}+15 t \frac{\partial}{\partial y}+\frac{\partial}{\partial u}-u \frac{\partial}{\partial v}, \\
\frac{\partial}{\partial x}+3 y \frac{\partial}{\partial y}+5 t \frac{\partial}{\partial t}-2 u \frac{\partial}{\partial u}-4 v \frac{\partial}{\partial v} .
\end{gathered}
$$

2.1. Symmetry Reduction of $(2 a)$ and $(2 b)$. One of the main reasons for calculating symmetries of a differential equation is to use them for obtaining symmetry reductions and finding exact solutions. This can be achieved with the use of Lie point symmetries admitted by (2a) and (2b). It is well known fact that the reduction of a partial differential equation with respect to $r$-dimensional (solvable) subalgebra of its Lie symmetry algebra leads to reducing the number of independent variables by $r$.

Consider the first three translation symmetries and let $\Gamma=$ $\Gamma_{1}+\Gamma_{2}+\Gamma_{3}$. We use $\Gamma$ to reduce $(2 \mathrm{a})$ and $(2 \mathrm{~b})$ to a system of partial differential equations (PDEs) in two independent variables. The symmetry $\Gamma$ yields the following invariants:

$$
\begin{gathered}
f=t-y, \\
g=x-y, \\
\phi=u, \\
\psi=v .
\end{gathered}
$$

Considering $\phi, \psi$ as the new dependent variables and $f$ and $g$ as new independent variables, (2a) and (2b) transforms to

$$
\begin{gathered}
\phi_{g g g g g}+5 \phi_{g} \phi^{2}-5 \phi_{f g g}+5 \phi_{g} \psi+5 \psi_{f}+\phi_{f}+5 \psi_{g} \phi \\
+\frac{25}{2} \phi_{g} \phi_{g g}+5 \psi_{g}+5 \phi_{g g g} \phi-5 \phi_{g g g}=0 \\
\phi_{f}+\phi_{g}+\psi_{g}=0,
\end{gathered}
$$

which is a system of nonlinear PDEs in two independent variables $f$ and $g$. We now further reduce this system using its symmetries. This system has the two translation symmetries, namely,

$$
\Upsilon_{1}=\frac{\partial}{\partial f}, \quad \Upsilon_{2}=\frac{\partial}{\partial g} .
$$


By taking a linear combination $\rho \Upsilon_{1}+\Upsilon_{2}$ of the above symmetries, we see that it yields the invariants

$$
z=f-\rho g, \quad \phi=F, \quad \psi=G
$$

Now treating $F$ and $G$ as new dependent variables and $z$ as the new independent variable the above system transforms to the following system of nonlinear coupled ODEs:

$$
\begin{aligned}
& \rho^{5} F^{\prime \prime \prime \prime \prime}(z)+5 \rho^{3} F(z) F^{\prime \prime \prime}(z)-5 \rho^{3} F^{\prime \prime \prime}(z) \\
& \quad+5 \rho^{2} F^{\prime \prime \prime}(z)+5 \rho G(z) F^{\prime}(z)+5 \rho F(z)^{2} F^{\prime}(z) \\
& \quad-F^{\prime}(z)+\frac{25}{2} \rho^{3} F^{\prime}(z) F^{\prime \prime}(z)+5 \rho F(z) G^{\prime}(z) \\
& \quad+5 \rho G^{\prime}(z)-5 G^{\prime}(z)=0, \\
& \rho F^{\prime}(z)-F^{\prime}(z)+\rho G^{\prime}(z)=0 .
\end{aligned}
$$

2.2. Exact Solutions Using the Extended tanh Method. In this section we use the extended tanh function method which was introduced by Wazwaz [7]. The basic idea in this method is to assume that the solution of (10a) and (10b) can be written in the form

$$
\begin{aligned}
& F(z)=\sum_{i=-M}^{M} A_{i} H(z)^{i}, \\
& G(z)=\sum_{i=-M}^{M} B_{i} H(z)^{i},
\end{aligned}
$$

where $H(z)$ satisfies an auxiliary equation, say for example the Riccati equation

$$
H^{\prime}(z)=1-H^{2}(z)
$$

whose solution is given by

$$
H(z)=\tanh (z)
$$

The positive integer $M$ will be determined by the homogeneous balance method between the highest order derivative and highest order nonlinear term appearing in (10a) and (10b). $A_{i}, B_{i}$ are parameters to be determined.

In our case, the balancing procedure gives $M=2$ and so the solutions of (10a) and (10b) are of the form

$$
\begin{gathered}
F(z)=A_{-2} H^{-2}+A_{-1} H^{-1}+A_{0}+A_{1} H+A_{2} H^{2}, \\
G(z)=B_{-2} H^{-2}+B_{-1} H^{-1}+B_{0}+B_{1} H+B_{2} H^{2} .
\end{gathered}
$$

Substituting (13a) and (13b) into (10a) and (10b) and making use of the Riccati equation (11) and then equating the coefficients of the functions $H^{i}$ to zero, we obtain an algebraic system of equations in terms of $A_{i}$ and $B_{i}(i=-2,-1,0,1,2)$.

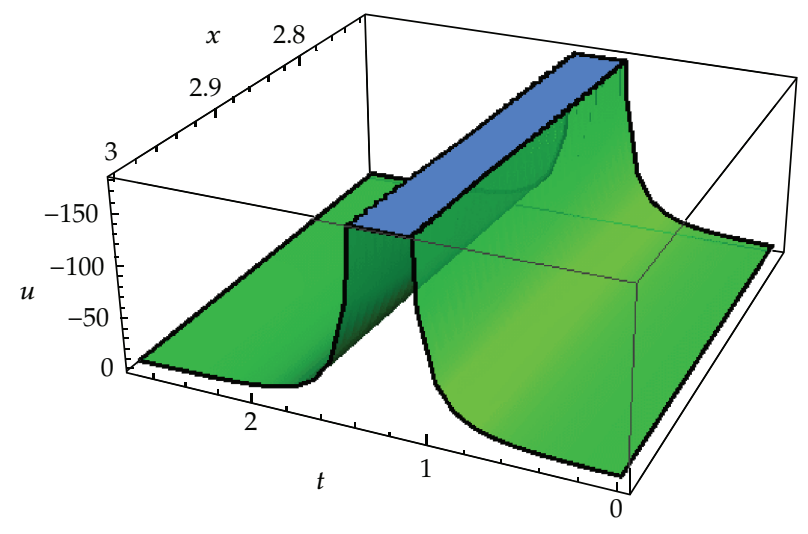

FIGURE 1: Evolution of travelling wave solution (14) with parameters $y=0, \rho=0.42$.

Solving the resultant system of algebraic equations, with the aid of Mathematica, one possible set of values of $A_{i}$ and $B_{i}(i=-2,-1,0,1,2)$ is

$$
\begin{gathered}
A_{-2}=-24 \rho^{2}, \\
A_{-1}=0, \\
A_{0}=\frac{-1+\rho+16 \rho^{3}}{\rho}, \\
A_{1}=0, \\
A_{2}=-24 \rho^{2}, \\
B_{-2}=24 \rho(-1+\rho), \\
B_{-1}=0, \\
B_{0}=-\frac{-5+9 \rho-80 \rho^{3}-5 \rho^{2}+80 \rho^{4}+2816 \rho^{6}}{5 \rho^{2}}, \\
B_{1}=0, \\
B_{2}=24 \rho(-1+\rho),
\end{gathered}
$$

where $\rho$ is any root of $2816 \rho^{6}+320 \rho^{4}-320 \rho^{3}-5 \rho^{2}+9 \rho-5$. As a result, a solution of (1) is

$$
\begin{aligned}
u(t, x, y)= & A_{-2} \operatorname{coth}^{2}(z)+A_{-1} \operatorname{coth}(z)+A_{0} \\
& +A_{1} \tanh (z)+A_{2} \tanh ^{2}(z),
\end{aligned}
$$

where $z=t-\rho x+(\rho-1) y$.

A profile of the solution (14) is given in Figure 1.

2.3. Exact Solutions Using Extended Jacobi Elliptic Function Method. In this subsection we obtain exact solutions of (1) in terms of the Jacobi elliptic functions. We note that the cosine-amplitude function, $\operatorname{cn}(z \mid \omega)$, and the sine-amplitude 
function, $\operatorname{sn}(z \mid \omega)$ are solutions of the first-order differential equations

$$
\begin{gathered}
H^{\prime}(z)=-\left\{\left(1-H^{2}(z)\right)\left(1-\omega+\omega H^{2}(z)\right)\right\}^{1 / 2}, \\
H^{\prime}(z)=\left\{\left(1-H^{2}(z)\right)\left(1-\omega H^{2}(z)\right)\right\}^{1 / 2},
\end{gathered}
$$

respectively [24]. We recall the following facts.

(i) When $\omega \rightarrow 1$, the Jacobi elliptic functions degenerate to the hyperbolic functions, $\operatorname{cn}(z \mid \omega) \rightarrow$ $\operatorname{sech}(z), \operatorname{sn}(z \mid \omega) \rightarrow \tanh (z)$.

(ii) When $\omega \rightarrow 0$, the Jacobi elliptic functions degenerate to the trigonometric functions, $\operatorname{cn}(z \quad \mid \quad \omega) \rightarrow$ $\cos (z), \operatorname{sn}(z \mid \omega) \rightarrow \sin (z)$.

(iii) $\operatorname{nc}(z \mid \omega)=1 / \operatorname{cn}(z \mid \omega)$, $\operatorname{ns}(z \mid \omega)=1 / \operatorname{sn}(z \mid \omega)$.

We now treat the above ODEs as our auxillary equations and apply the procedure of the previous subsection to system (10a) and (10b). Leaving out the details, we obtain two solutions, the cnoidal and snoidal wave solutions, corresponding to the two equations (15) and (16) given by, respectively,

$$
\begin{aligned}
u(t, x, y)= & A_{-2} \mathrm{nc}^{2}(z \mid \omega)+A_{-1} \mathrm{nc}(z \mid \omega) \\
& +A_{0}+A_{1} \mathrm{cn}(z \mid \omega)+A_{2} \mathrm{cn}^{2}(z \mid \omega),
\end{aligned}
$$

where

$$
\begin{gathered}
A_{-2}=-3 \rho^{2}+3 \rho^{2} \omega, \\
A_{-1}=0, \\
A_{0}=-\frac{2 \rho^{3} \omega+1-\rho-\rho^{3}}{\rho}, \\
A_{1}=0, \\
A_{2}=3 \rho^{2} \omega, \\
B_{-2}=-3 \rho(\omega-1)(\rho-1), \\
B_{-1}=0, \\
-\frac{-5+9 \rho-5 \rho^{2}-5 \rho^{3}+\rho^{6}-10 \rho^{4} \omega}{5 \rho^{2}} \\
+\frac{16 \rho^{6} \omega^{2}-16 \rho^{6} \omega+10 \rho^{3} \omega+5 \rho^{4}}{5 \rho^{2}}, \\
B_{1}=0, \\
B_{2}=-3 \rho \omega(\rho-1),
\end{gathered}
$$

with $\rho$ as any root of $\left(16 \omega^{2}-16 \omega+1\right) \rho^{6}+(5-10 \omega) \rho^{4}+(10 \omega-$ 5) $\rho^{3}-5 \rho^{2}+9 \rho-5$ and

$$
\begin{aligned}
u(t, x, y)= & A_{-2} \mathrm{~ns}^{2}(z \mid \omega)+A_{-1} \mathrm{~ns}(z \mid \omega)+A_{0} \\
& +A_{1} \mathrm{sn}(z \mid \omega)+A_{2} \mathrm{sn}^{2}(z \mid \omega)
\end{aligned}
$$

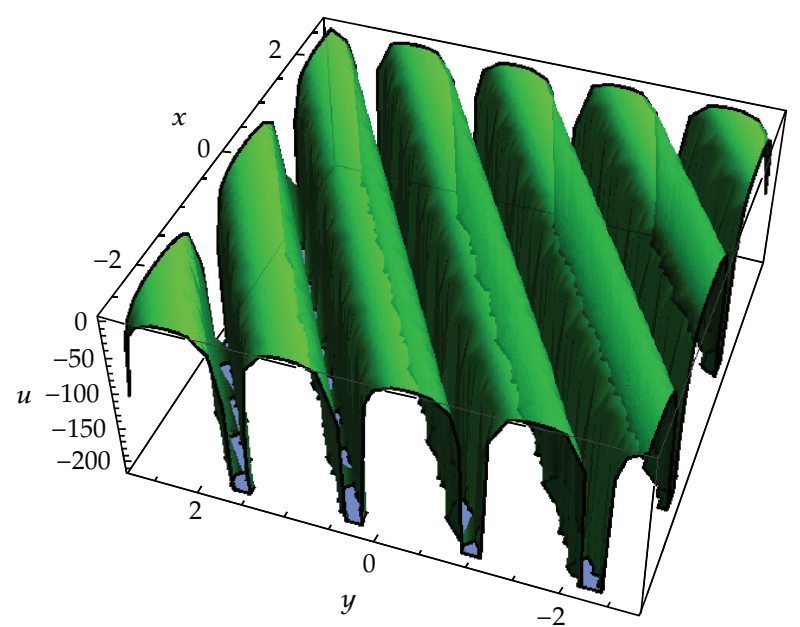

FIGURE 2: Evolution of travelling wave solution (17) with parameters $t=0, \omega=0.1, \quad \rho=-1.53$.

with

$$
\begin{gathered}
A_{-2}=-3 \rho^{2}, \\
A_{-1}=0,
\end{gathered}
$$

$$
A_{0}=\frac{-1+\rho+\rho^{3}+\rho^{3} \omega}{\rho}
$$$$
A_{1}=0 \text {, }
$$$$
A_{2}=-3 \omega \rho^{2} \text {, }
$$$$
B_{-2}=3 \rho(-1+\rho) \text {, }
$$$$
B_{-1}=0 \text {, }
$$

$$
\begin{gathered}
B_{0}=-\frac{-5+9 \rho-5 \rho^{2}-5 \rho^{3}+\rho^{6} \omega^{2}}{5 \rho^{2}} \\
+\frac{14 \rho^{6} \omega-5 \rho^{3} \omega+5 \rho^{4} \omega+5 \rho^{4}+\rho^{6}}{5 \rho^{2}}, \\
B_{1}=0, \\
B_{2}=3 \omega \rho(-1+\rho),
\end{gathered}
$$

where $\rho$ is any root of $\left(\omega^{2}+14 \omega+1\right) \rho^{6}+(5 \omega+5) \rho^{4}-(5 \omega+$ 5) $\rho^{3}-5 \rho^{2}+9 \rho-5$ and $z=t-\rho x+(\rho-1) y$.

A profile of solutions (17), (19) is given in Figures 2 and 3.

\section{Conservation Laws}

In this section we construct conservation laws for (2a) and (2b). The multiplier method will be used [23]. We first recall some basic results that will be used later in this section. 


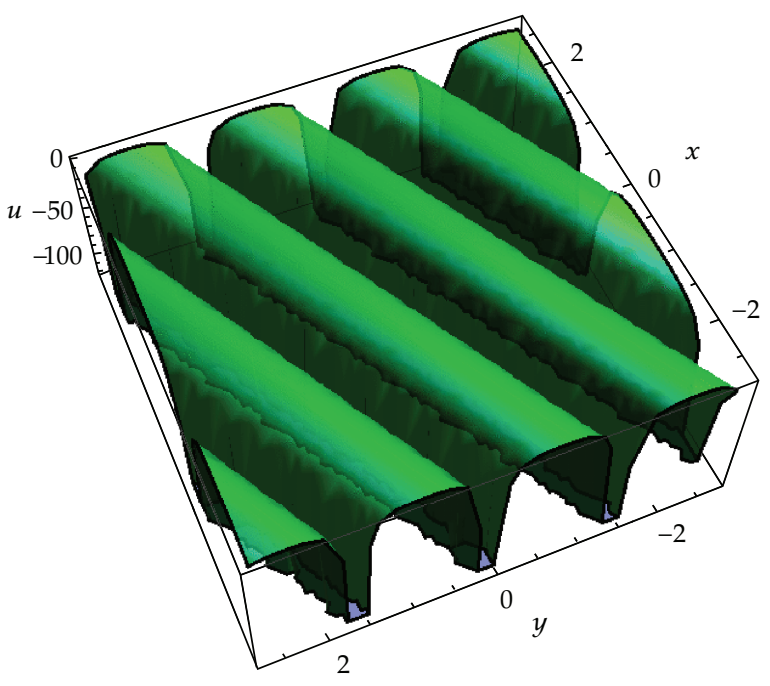

FIGURE 3: Evolution of travelling wave solution (19) with parameters $t=0, \omega=0.2, \rho=-1.12$.

Consider a $k$ th-order system of PDEs of $n$ independent variables $x=\left(x^{1}, x^{2}, \ldots, x^{n}\right)$ and $m$ dependent variables $u=$ $\left(u^{1}, u^{2}, \ldots, u^{m}\right)$, namely,

$$
E_{\alpha}\left(x, u, u_{(1)}, \ldots, u_{(k)}\right)=0, \quad \alpha=1, \ldots, m,
$$

where $u_{(1)}, u_{(2)}, \ldots, u_{(k)}$ denote the collections of all first, second,..., $k$ th-order partial derivatives, that is, $u_{i}^{\alpha}=$ $D_{i}\left(u^{\alpha}\right), u_{i j}^{\alpha}=D_{j} D_{i}\left(u^{\alpha}\right), \ldots$, respectively, with the total derivative operator with respect to $x^{i}$ given by

$$
D_{i}=\frac{\partial}{\partial x^{i}}+u_{i}^{\alpha} \frac{\partial}{\partial u^{\alpha}}+u_{i j}^{\alpha} \frac{\partial}{\partial u_{j}^{\alpha}}+\cdots, \quad i=1, \ldots, n,
$$

where the summation convention is used whenever appropriate [13]. We recall that the Euler-Lagrange operator, for each $\alpha$, is given by

$$
\frac{\delta}{\delta u^{\alpha}}=\frac{\partial}{\partial u^{\alpha}}+\sum_{s \geq 1}(-1)^{s} D_{i_{1}} \cdots D_{i_{s}} \frac{\partial}{\partial u_{i_{1} i_{2} \cdots i_{s}}^{\alpha}}, \quad \alpha=1, \ldots, m .
$$

The $n$-tuple vector $T=\left(T^{1}, T^{2}, \ldots, T^{n}\right), T^{j} \in \mathscr{A}$, (space of differential functions) $j=1, \ldots, n$, is a conserved vector of (21) if $T^{i}$ satisfies

$$
\left.D_{i} T^{i}\right|_{(3.1)}=0 .
$$

A multiplier $\Lambda_{\alpha}\left(x, u, u_{(1)}, \ldots\right)$ has the property that

$$
\Lambda_{\alpha} E_{\alpha}=D_{i} T^{i}
$$

hold identically. Here we will consider multipliers of the zeroth order, that is, $\Lambda_{\alpha}=\Lambda_{\alpha}(t, x, y, u, v)$. The right hand side of (25) is a divergence expression. The determining equation for the multiplier $\Lambda_{\alpha}$ is given by

$$
\frac{\delta\left(\Lambda_{\alpha} E_{\alpha}\right)}{\delta u^{\alpha}}=0
$$

After calculating the multipliers one can obtain the conserved vectors via a homotopy formula [23].
3.1. Conservation Laws of (1). We now construct conservation laws for the two-dimensional integrable generalization of the Kaup-Kupershmidt equation (1) using the multiplier approach. For the coupled system (2a) and (2b), we obtain multipliers of the form, $\Lambda_{1}=\Lambda_{1}(t, x, y, u, v)$ and $\Lambda_{2}=$ $\Lambda_{2}(t, x, y, u, v)$ that are given by

$$
\begin{gathered}
\Lambda_{1}=y f_{2}(t)+f_{1}(t), \\
\Lambda_{2}=-5 x f_{2}(t)+f_{3}(t)-\frac{1}{2} y\left(y f_{2}^{\prime}(t)+2 f_{1}^{\prime}(t)\right),
\end{gathered}
$$

where $f_{i}, i=1,2,3$ are arbitrary functions of $t$. Corresponding to the above multipliers we obtain the following nonlocal conserved vector of (1):

$$
\begin{aligned}
& T^{t}=f_{1}(t) u+y f_{2}(t) u, \\
& T^{x}=\frac{1}{12}\left\{60 f_{1}(t) u_{x x} u+60 y f_{2}(t) u_{x x} u\right. \\
& +60 f_{1}(t) u \int u_{y} d x+60 y f_{2}(t) u \int u_{y} d x \\
& +20 f_{1}(t) u^{3}+20 y f_{2}(t) u^{3}+45 y f_{2}(t) u_{x}{ }^{2} \\
& +40 f_{1}(t) u_{x y}+40 y f_{2}(t) u_{x y} \\
& +12 y f_{2}(t) u_{x x x x}+60 x f_{2}(t) \int u_{y} d x \\
& -12 f_{3}(t) \int u_{y} d x+45 f_{1}(t) u_{x}^{2} \\
& -20 f_{2}(t) u_{x}+12 f_{1}(t) u_{x x x x} \\
& \left.+6 y^{2} f_{2}^{\prime}(t) \int u_{y} d x+12 y f_{1}^{\prime}(t) \int u_{y} d x\right\}, \\
& T^{y}=\frac{1}{6}\left\{-3 y^{2} f_{2}^{\prime}(t) u-6 y f_{1}^{\prime}(t) u\right. \\
& -30 x f_{2}(t) u+6 f_{3}(t) u \\
& +10 y f_{2}(t) u_{x x}-30 y f_{2}(t) \int u_{y} d x \\
& \left.-30 f_{1}(t) \int u_{y} d x+10 f_{1}(t) u_{x x}\right\} .
\end{aligned}
$$

Remark 1. Due to the presence of the arbitrary functions, $f_{i}$, $i=1,2,3$, in the multipliers, one can obtain an infinitely many nonlocal conservation laws.

\section{Concluding Remarks}

In this paper we studied the two-dimensional generalization of the Kaup-Kupershmidt equation (1). Lie point symmetries of this equation were obtained and the three translation symmetries were used to transform the equation into a system of ODEs. Then the extended tanh method and the extended Jacobi elliptic function method were employed to solve this ODEs system to obtain exact solutions of (1). Furthermore, conservation laws of (1) were also computed using the 
multiplier approach. The conservation laws consisted of an infinite number of nonlocal conserved vectors.

\section{Acknowledgments}

A. R. Adem and C. M. Khalique would like to thank the Organizing Committee of "Symmetries, Differential Equations, and Applications: Galois Bicentenary" (SDEA2012) Conference for their kind hospitality during the conference.

\section{References}

[1] M. J. Ablowitz and P. A. Clarkson, Solitons, Nonlinear Evolution Equations and Inverse Scattering, Cambridge University Press, Cambridge, UK, 1991.

[2] R. Hirota, "Exact solution of the korteweg-de vries equation for multiple collisions of solitons," Physical Review Letters, vol. 27, no. 18, pp. 1192-1194, 1971.

[3] M. L. Wang, "Solitary wave solutions for variant Boussinesq equations," Physics Letters A, vol. 199, no. 3-4, pp. 169-172, 1995.

[4] Sirendaoreji and S. Jiong, "Auxiliary equation method for solving nonlinear partial differential equations," Physics Letters A, vol. 309, no. 5-6, pp. 387-396, 2003.

[5] G. B. Zheng, B. Liu, Z. J. Wang, and S. K. Zheng, "Variational principle for nonlinear magneto-electroe-lastodynamics with finite displacement by He's semi-inverse method," International Journal of Nonlinear Sciences and Numerical Simulation, vol. 10, no. 11-12, pp. 1523-1526, 2009.

[6] A. M. Wazwaz, "The tanh and the sine-cosine methods for compact and noncompact solutions of the nonlinear KleinGordon equation," Applied Mathematics and Computation, vol. 167, no. 2, pp. 1179-1195, 2005.

[7] A. M. Wazwaz, "New solitary wave solutions to the KuramotoSivashinsky and the Kawahara equations," Applied Mathematics and Computation, vol. 182, no. 2, pp. 1642-1650, 2006.

[8] P. J. Olver, Applications of Lie Groups to Differential Equations, vol. 107 of Graduate Texts in Mathematics, Springer, Berlin, Germany, 2nd edition, 1993.

[9] M. Jimbo and T. Miwa, "Solitons and infinite dimensional lie algebras," Publications of the RIMS, Kyoto University, vol. 19, no. 3, pp. 943-1001, 1983.

[10] B. G. Konopelchenko and V. G. Dubrovsky, "Some new integrable nonlinear evolution equations in $(2+1)$ dimensions," Physics Letters A, vol. 102, no. 1-2, pp. 15-17, 1984.

[11] V. G. Dubrovsky and Y. V. Lisitsyn, "The construction of exact solutions of two-dimensional integrable generalizations of Kaup-Kuperschmidt and Sawada-Kotera equations via $\partial$ dressing method," Physics Letters A, vol. 295, no. 4, pp. 198-207, 2002.

[12] V. G. Dubrovsky, A. V. Topovsky, and M. Y. Basalaev, "New exact solutions of two-dimensional integrable equations using the d-dressing method," Theoretical and Mathematical Physics, vol. 167, no. 3, pp. 725-739, 2011.

[13] N. H. Ibragimov, CRC Handbook of Lie Group Analysis of Differential Equations, vol. 1-3, CRC Press, Boca Raton, Fla,USA, 1994-1996.

[14] G. W. Bluman and S. Kumei, Symmetries and Differential Equations, vol. 81 of Applied Mathematical Sciences, Springer, New York, NY, USA, 1989.
[15] P. D. Lax, "Integrals of nonlinear equations of evolution and solitary waves," Communications on Pure and Applied Mathematics, vol. 21, pp. 467-490, 1968.

[16] T. B. Benjamin, "The stability of solitary waves," Proceedings of the Royal Society, vol. 328, no. 1573, pp. 153-183, 1972.

[17] R. J. Knops and C. A. Stuart, "Quasiconvexity and uniqueness of equilibrium solutions in nonlinear elasticity," Archive for Rational Mechanics and Analysis, vol. 86, no. 3, pp. 233-249, 1984.

[18] R. J. LeVeque, Numerical Methods for Conservation Laws, Birkhäuser, Basel, Switzerland, 1992.

[19] E. Godlewski and P. A. Raviart, Numerical Approximation of Hyperbolic Systems of Conservation Laws, Springer, Berlin, Germany, 1996.

[20] A. Sjöberg, "Double reduction of PDEs from the association of symmetries with conservation laws with applications," Applied Mathematics and Computation, vol. 184, no. 2, pp. 608-616, 2007.

[21] A. H. Bokhari, A. Y. Al-Dweik, A. H. Kara, F. M. Mahomed, and F. D. Zaman, "Double reduction of a nonlinear $(2+1)$ wave equation via conservation laws," Communications in Nonlinear Science and Numerical Simulation, vol. 16, no. 3, pp. 1244-1253, 2011.

[22] G. L. Caraffini and M. Galvani, "Symmetries and exact solutions via conservation laws for some partial differential equations of mathematical physics," Applied Mathematics and Computation, vol. 219, no. 4, pp. 1474-1484, 2012.

[23] S. C. Anco and G. W. Bluman, "Direct construction method for conservation laws of partial differential equations. Part I: examples of conservation law classifications," European Journal of Applied Mathematics, vol. 13, no. 5, pp. 545-566, 2002.

[24] I. S. Gradshteyn and I. M. Ryzhik, Table of Integrals, Series, and Products, Academic Press, New York, NY, USA, 7th edition, 2007. 


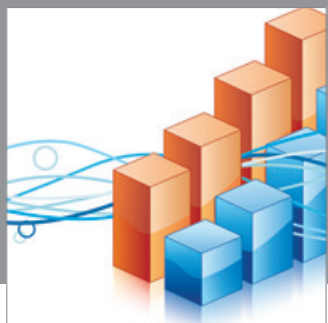

Advances in

Operations Research

mansans

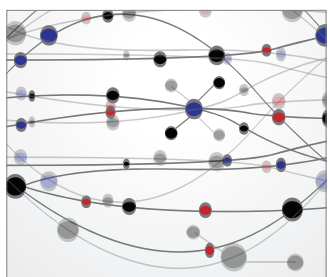

The Scientific World Journal
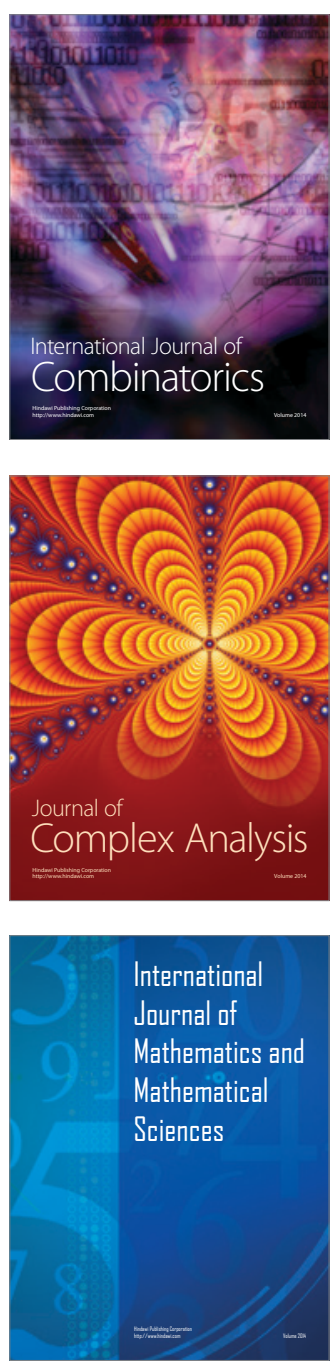
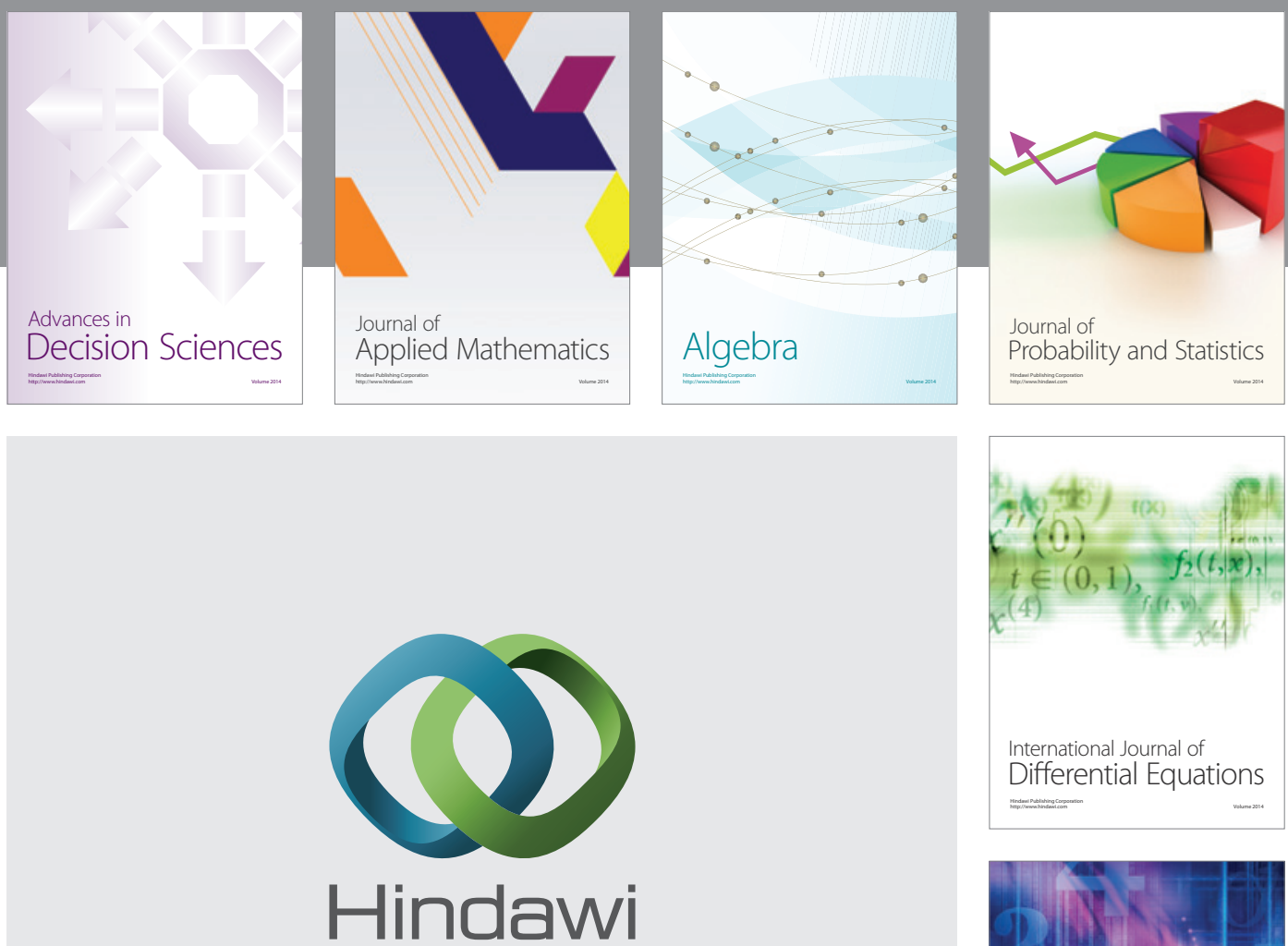

Submit your manuscripts at http://www.hindawi.com
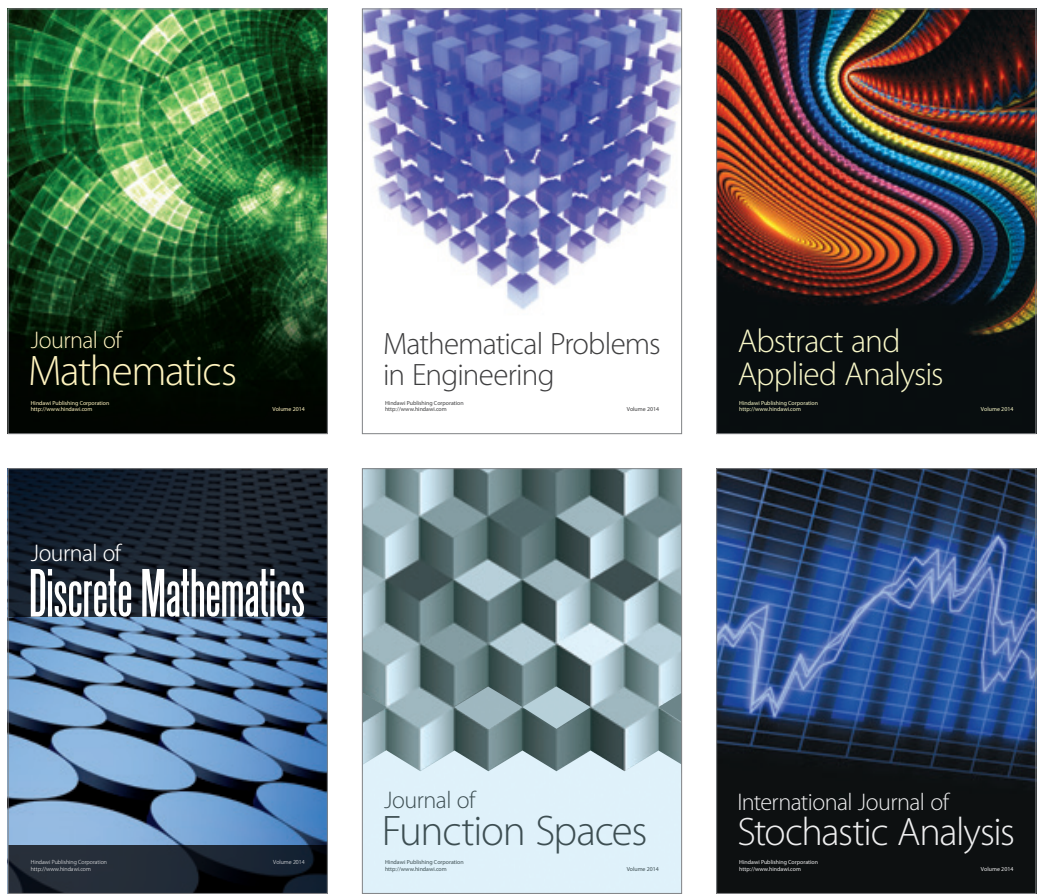

Journal of

Function Spaces

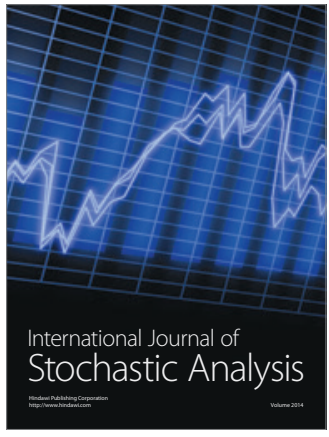

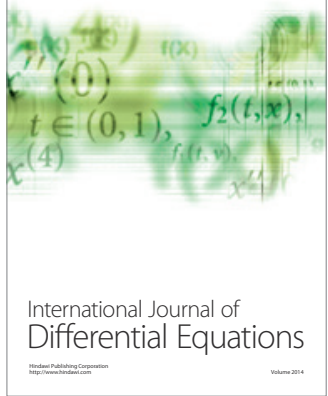
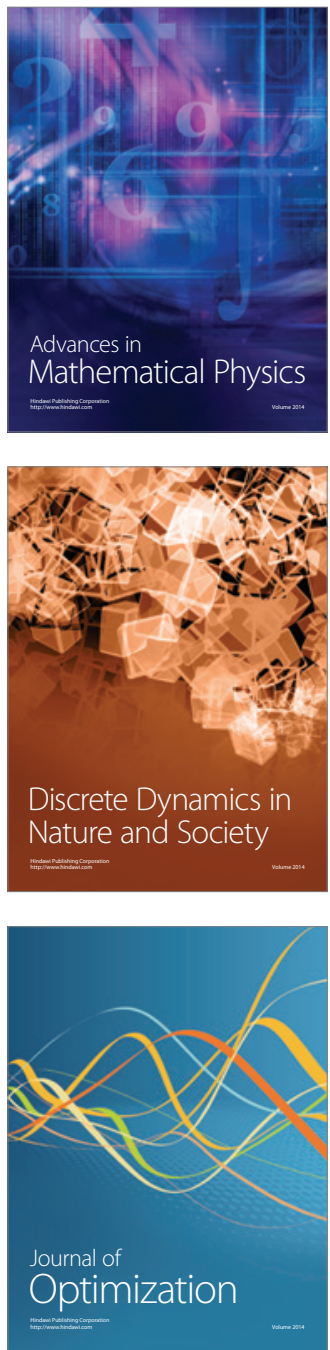